Article

\title{
Some Iterative Properties of $\left(\mathcal{F}_{1}, \mathcal{F}_{2}\right)$-Chaos in Non-Autonomous Discrete Systems
}

\author{
Xiao Tang ${ }^{1}$, Guanrong Chen ${ }^{2}$ and Tianxiu $\mathrm{Lu}^{3, *}$ \\ 1 School of Mathematical Sciences, Sichuan Normal University, Chengdu 610068, China; 80651177@163.com \\ 2 Department of Electronic Engineering, City University of Hong Kong, Hong Kong SAR 999077, China; \\ eegchen@cityu.edu.hk \\ 3 School of Mathematics and Statistics, Sichuan University of Science and Engineering, Zigong 643000, China \\ * Correspondence: lubeeltx@163.com; Tel.: +86-813-2105950
}

Received: 24 January 2018; Accepted: 7 March 2018; Published: 12 March 2018

\begin{abstract}
This paper is concerned with invariance $\left(\mathcal{F}_{1}, \mathcal{F}_{2}\right)$-scrambled sets under iterations. The main results are an extension of the compound invariance of Li-Yorke chaos and distributional chaos. New definitions of $\left(\mathcal{F}_{1}, \mathcal{F}_{2}\right)$-scrambled sets in non-autonomous discrete systems are given. For a positive integer $k$, the properties $P(k)$ and $Q(k)$ of Furstenberg families are introduced. It is shown that, for any positive integer $k$, for any $s \in[0,1]$, Furstenberg family $\bar{M}(s)$ has properties $P(k)$ and $Q(k)$, where $\bar{M}(s)$ denotes the family of all infinite subsets of $\mathbb{Z}^{+}$whose upper density is not less than $s$. Then, the following conclusion is obtained. $D$ is an $(\bar{M}(s), \bar{M}(t))$-scrambled set of $\left(X, f_{1, \infty}\right)$ if and only if $D$ is an $(\bar{M}(s), \bar{M}(t))$-scrambled set of $\left(X, f_{1, \infty}^{[m]}\right)$.
\end{abstract}

Keywords: nonautonomous discrete system; Furstenberg family; scrambled sets; chaos

MSC2010: 37B55, 37D45, 54H20

\section{Introduction}

Chaotic properties of a dynamical system have been extensively discussed since the introduction of the term chaos by Li and Yorke in 1975 [1] and Devaney in 1989 [2]. To describe some kind of unpredictability in the evolution of a dynamical system, other definitions of chaos have also been proposed, such as generic chaos [3], dense chaos [4], Li-Yorke sensitivity [5], and so on. An important generalization of Li-Yorke chaos is distributional chaos, which is given in 1994 by B. Schweizer and J. Smítal [6]. Then, theories related to scrambled sets are discussed extensively (see [7-12] and others). In 1997, the Furstenberg family was introduced by E. Akin [13]. J. Xiong, F. Tan described chaos with a couple of Furstenberg Families. $\left(\mathcal{F}_{1}, \mathcal{F}_{2}\right)$-chaos has also been defined [14]. Moreover, $\mathcal{F}$-sensitivity was given in [15] and shadowing properties were discussed in [16]. Most existing papers studied the chaoticity in autonomous discrete systems $(X, f)$. However, if a sequence of perturbations to a system are described by different functions, then there are a sequence of maps to describe them, giving rise to non-autonomous systems. Non-autonomous discrete systems were precisely introduced in [17], in connection with non-autonomous difference equations (see $[18,19]$ and some references therein).

Let $(X, \rho)$ (briefly, $X)$ be a compact metric space and consider a sequence of continuous maps $f_{n}: X \rightarrow X, n \in \mathbb{N}$, denoted by $f_{1, \infty}=\left(f_{1}, f_{2}, \cdots\right)$. This sequence defines a non-autonomous discrete system $\left(X, f_{1, \infty}\right)$. The orbit of any point $x \in X$ is given by the sequence $\left(f_{1}^{n}(x)\right)=\operatorname{Orb}\left(x, f_{1, \infty}\right)$, where $f_{1}^{n}=f_{n} \circ \cdots \circ f_{1}$ for $n \geq 1$, and $f_{1}^{0}$ is the identity map.

For $m \in \mathbb{N}$, define

$$
g_{1}=f_{m} \circ \cdots \circ f_{1}, g_{2}=f_{2 m} \circ \cdots \circ f_{m+1}, \ldots, g_{p}=f_{p m} \circ \cdots \circ f_{(p-1) m+1}, \ldots
$$


Call $\left(X, g_{1, \infty}\right)$ a compound system of $\left(X, f_{1, \infty}\right)$.

Also, denote $g_{1, \infty}$ by $f_{1, \infty}^{[m]}$ and denote $f_{n}^{k}=f_{n+k-1} \circ \cdots \circ f_{n}$ for $n \geq 1$. By [5], if $\left(f_{n}\right)_{n=1}^{\infty}$ converges uniformly to a map $f$. Then, for any $m \geq 2(m \in \mathbb{N})$, the sequence $\left(f_{n}^{n+m-1}\right)_{n=1}^{\infty}$ converges uniformly to $f^{m}$.

In the present work, some notions relating to Furstenberg families and properties $P(k), Q(k)$ are recalled in Sections 2 and 3. Section 4 states some definitions about $\left(\mathcal{F}_{1}, \mathcal{F}_{2}\right)$-chaos. In Section 5 , it is proved that, under the conditions of property $P(k)$ and positive shift-invariant, $f_{1, \infty}$ is $\left(\mathcal{F}_{1}, \mathcal{F}_{2}\right)$-chaos (strong $\left(\mathcal{F}_{1}, \mathcal{F}_{2}\right)$-chaos, strong $\mathcal{F}$-chaos) implies $f_{1, \infty}^{[k]}\left(k \in \mathbb{Z}^{+}\right)$is $\left(\mathcal{F}_{1}, \mathcal{F}_{2}\right)$-chaos (strong $\left(\mathcal{F}_{1}, \mathcal{F}_{2}\right)$-chaos, strong $\mathcal{F}$-chaos). If the conditions property $Q(k)$ and negative shift-invariant both hold, the above conclusion can be inversed. As a conclusion, for arbitrary $s$ and $t$ in $[0,1]$, for every $k \in \mathbb{Z}^{+}, f_{1, \infty}$ and $f_{1, \infty}^{[k]}$ can share the same $(\bar{M}(s), \bar{M}(t))$-scrambled set (Theorem 3 ).

In this paper, it is always assumed that all the maps $f_{n}, n \in \mathbb{N}$, are surjective. It should be noted that this condition is needed by most papers dealing with this kind of system (for example, [20-23]). It is assumed that sequence $\left(f_{n}\right)_{n=1}^{\infty}$ converges uniformly. The aim of this paper is to investigate the $\left(\mathcal{F}_{1}, \mathcal{F}_{2}\right)$-scrambled sets of $f_{1, \infty}$.

\section{Furstenberg Families}

Let $\mathcal{P}$ be the collection of all subsets of the positive integers set $\mathbb{Z}^{+}=\{0,1,2, \ldots\}$. A collection $\mathcal{F} \subset \mathcal{P}$ is called a Furstenberg family if it is hereditary upwards, i.e., $F_{1} \subset F_{2}$ and $F_{1} \in \mathcal{F}$ imply $F_{2} \in \mathcal{F}$. Obviously, the collection of all infinite subsets of $\mathbb{Z}^{+}$is a Furstenberg family, denoted by $\mathcal{B}$.

Define the dual family $k \mathcal{F}$ of a Furstenberg family $\mathcal{F}$ by

$$
k \mathcal{F}=\left\{F \in \mathcal{P}: \mathbb{Z}^{+}-F \notin \mathcal{F}\right\}=\left\{F \in \mathcal{P}: F \cap F^{\prime} \neq \phi \text { for any } F^{\prime} \in \mathcal{F}\right\} .
$$

It is clear that $k \mathcal{F}$ is a Furstenberg family and $k(k \mathcal{F})=\mathcal{F}$ (see [13]).

For $F \in \mathcal{P}, i \in \mathbb{Z}^{+}$, let $F-i=\{j-i \geq 0: j \in F\}$ and $F+i=\{j+i \geq 0: j \in F\}$. Furstenberg family $\mathcal{F}$ is positive shift-invariant if $F+i \in \mathcal{F}$ for every $F \in \mathcal{F}$ and any $i \in \mathbb{Z}^{+}$. Furstenberg family $\mathcal{F}$ is negative shift-invariant if $F-i \in \mathcal{F}$ for every $F \in \mathcal{F}$ and any $i \in \mathbb{Z}^{+}$. Furstenberg family $\mathcal{F}$ is shift-invariant if it is positive shift-invariant and negative shift-invariant.

The following shows a class of Furstenberg families which is related to upper density.

Let $F \subset \mathcal{P}$. The upper density and the lower density of $F$ are defined as follows:

$$
\bar{\mu}(F)=\limsup _{n \rightarrow \infty} \frac{\#(F \cap\{0,1, \ldots, n-1\})}{n}, \underline{\mu}(F)=\liminf _{n \rightarrow \infty} \frac{\#(F \cap\{0,1, \ldots, n-1\})}{n},
$$

where \# $(A)$ denotes the cardinality of the set $A$.

For any $s$ in $[0,1]$, set $\bar{M}(s)=\{F \in \mathcal{B}: \bar{\mu}(F) \geq s\}$.

Proposition 1. For any $\sin [0,1], \bar{M}(s)$ is shift-invariant Furstenberg family. And $\bar{M}(0)=\mathcal{B}$.

\section{Proof.}

(i) Let $F_{1}, F_{2} \in \bar{M}(s), F_{1} \subset F_{2}$, then, $\forall n \in \mathbb{N}$ (where $\mathbb{N}=\{1,2,3, \ldots\}$ ),

$$
\bar{\mu}\left(F_{1}\right)=\limsup _{n \rightarrow \infty} \frac{\#\left(F_{1} \cap\{0,1, \ldots, n-1\}\right)}{n} \leq \limsup _{n \rightarrow \infty} \frac{\#\left(F_{2} \cap\{0,1, \ldots, n-1\}\right)}{n}=\bar{\mu}\left(F_{2}\right)
$$

Thus, $F_{1} \in \bar{M}(s)$ (i.e., $\left.\bar{\mu}\left(F_{1}\right) \geq s\right)$ implies $F_{2} \in \bar{M}(s)$ (i.e., $\bar{\mu}\left(F_{1}\right) \geq s$ ). So, $\bar{M}(s)(\forall s \in[0,1])$ are Furstenberg families. 
(ii) Let $F \in \bar{M}(s)$, that is, $\bar{\mu}(F)=\limsup _{n \rightarrow \infty} \frac{\#(F \cap\{0,1, \ldots, n-1\})}{n} \geq s$. Denote $F=\left\{t_{1}, t_{2}, \cdots\right\}$ (where $t_{k} \in \mathbb{Z}^{+}, t_{k_{1}}<t_{k_{2}}\left(k_{1}<k_{2}\right)$ ), then $F+i=\left\{t_{1}+i, t_{2}+i, \cdots\right\}$ and $F-i=\left\{t_{k_{1}}-i, t_{k_{2}}-i, \cdots\right\}\left(t_{k_{j}}-i \geq 0\right)$ for any $i \in \mathbb{Z}^{+}$.

$$
\begin{gathered}
\limsup _{n \rightarrow \infty} \frac{\#((F+i) \cap\{0,1, \ldots, n-1\})}{n}=\limsup _{n \rightarrow \infty} \frac{\#\left(\left\{t_{1}+i, t_{2}+i, \cdots\right\} \cap\{0,1, \ldots, n-1\}\right)}{n} \\
=\limsup _{n \rightarrow \infty} \frac{\#\left(\left\{t_{1}, t_{2}, \cdots\right\} \cap\{0,1, \ldots, n-1\}\right)}{n}=\bar{\mu}(F) \geq s
\end{gathered}
$$

and

$$
\limsup _{n \rightarrow \infty} \frac{\#((F-i) \cap\{0,1, \ldots, n-1\})}{n} \geq \limsup _{n \rightarrow \infty} \frac{\#(F \cap\{0,1, \ldots, n-1\})-i}{n}=\bar{\mu}(F) \geq s
$$

So, $\bar{M}(s)$ is shift-invariant.

(iii) Obviously,

$$
\bar{M}(0)=\{F \in \mathcal{B}: \bar{\mu}(F) \geq 0\}=\left\{F \in \mathcal{B}: \limsup _{n \rightarrow \infty} \frac{\#(F \cap\{0,1, \ldots, n-1\})}{n} \geq 0\right\}=\mathcal{B} .
$$

This completes the proof.

\section{Properties $P(k), Q(k)$ of Furstenberg Families}

Definition 1. Let $k$ be a positive integer and $\mathcal{F}$ be a Furstenberg family.

(1) For any $F \in \mathcal{F}$, if there exists an integer $j \in\{0,1, \cdots, k-1\}$ such that $F_{k, j}=\left\{i \in \mathbb{Z}^{+}: k i+j \in F\right\} \in \mathcal{F}$, we say $\mathcal{F}$ have property $P(k)$;

(2) If $F_{k}=\left\{k i+j \in \mathbb{Z}^{+}: j \in\{0,1, \cdots, k-1\}, i \in F\right\} \in \mathcal{F}$, we say $\mathcal{F}$ have property $Q(k)$.

The following proposition is given by [24]. For completeness, we give the proofs.

Proposition 2. For any $s \in[0,1]$ and any $k \in \mathbb{Z}^{+}, \bar{M}(s)$ have properties $P(k)$ and $Q(k)$.

\section{Proof.}

(1) If $k=1, \forall F \in \bar{M}(s), F_{1,0}=\left\{i \in \mathbb{Z}^{+}: i \in F\right\}=F$, i.e., there exists an integer $j=0$ such that $F_{k, j} \in \bar{M}(s)$. The following will discuss the case $k>1$.

If $s=0, \bar{M}(0)=\mathcal{B} . \forall F \in \mathcal{B}, \forall k \in \mathbb{Z}^{+}$, obviously, there exist $j \in\{0,1, \ldots, k-1\}$ such that $F_{k, j} \in \mathcal{B}$.

If $0<s \leq 1$, suppose properties $P(k)$ does not hold. Then there exists a $F \in \bar{M}(s)$ such that $\bar{\mu}\left(F_{k, j}\right)<s$ for every $j \in\{0,1, \ldots, k-1\}$.

For any $j \in\{0,1, \ldots, k-1\}$, put $\varepsilon_{j}>0$ which satisfied $\bar{\mu}\left(F_{k, j}\right)<s-\varepsilon_{j}$. One can find a sufficiently large number $N$ such that, $n \geq N, \#_{n}\left(F_{k, j}\right)<n\left(s-\varepsilon_{j}\right)$ (where $\#_{n}\left(F_{k, j}\right)$ denotes the cardinality of the set $\left.F_{k, j} \cap\{0,1, \ldots, n-1\}\right)$. Then $\#_{n}\left(F_{k, j}^{c}\right)>n-n\left(s-\varepsilon_{j}\right)$, where $F_{k, j}^{c}$ denotes the complementary set of $F_{k, j}$.

Give an integer $m=k n+l_{m}>k N, l_{m} \in\{0,1, \ldots, k-1\}$. By the definition of $F_{k, j}, k i+j \notin F$ if $i \notin F_{k, j}$. And $k i_{1}+j_{1} \neq k i_{2}+j_{2}$ if $i_{1}, i_{2} \in\{0,1, \ldots, n-1\}, j_{1}, j_{2} \in\{0,1, \ldots, k-1\}$ and $j_{1} \neq j_{2}$. Then

$$
\#_{m}\left(F^{c}\right) \geq \sum_{j=0}^{k-1} \#_{n}\left(F_{k, j}^{c}\right)>\sum_{j=0}^{k-1}\left(n-n\left(s-\varepsilon_{j}\right)\right) .
$$


So,

$$
\#_{m}(F)<m-\sum_{j=0}^{k-1}\left(n-n\left(s-\varepsilon_{j}\right)\right) .
$$

Put $\varepsilon=\min \left\{\varepsilon_{j}: j=0,1, \ldots, k-1\right\}$, then

$$
\begin{gathered}
\bar{\mu}(F)=\limsup _{n \rightarrow \infty} \frac{\#_{m}(F)}{m} \leq \lim _{n \rightarrow \infty} \frac{m-\sum_{j=0}^{k-1}\left(n-n\left(s-\varepsilon_{j}\right)\right)}{m} \leq \lim _{n \rightarrow \infty} \frac{m-k(n-n(s-\varepsilon))}{m} \\
=\lim _{n \rightarrow \infty} \frac{k n+l_{m}-k n+k n(s-\varepsilon)}{k n+l_{m}}=s-\varepsilon<s
\end{gathered}
$$

This contradicts to $\bar{\mu}(F) \geq s$.

(2) Similarly, just consider the case $k>1,0<s \leq 1$.

Suppose properties $Q(k)$ does not hold. Then there exists an integer $F \in \bar{M}(s)$ such that $\bar{\mu}\left(F_{k}\right)<s$. Put $\varepsilon>0$ which satisfied $\bar{\mu}\left(F_{k}\right)<s-\varepsilon$. One can find a sufficiently large number $N$ such that, $m \geq N, \#_{m}\left(F_{k}\right)<m(s-\varepsilon)$. Give a $m=k n+l_{m}>k N(m \geq N), l_{m} \in\{0,1, \ldots, k-1\}$. By the definition of $F_{k}, k i+j \in F_{k}(j \in\{0,1, \ldots, k-1\})$ if $i \in F$. And $k i_{1}+j_{1} \neq k i_{2}+j_{2}$ if $i_{1} \neq i_{2}$ and $j_{1}, j_{2} \in\{0,1, \ldots, k-1\}$. Then

$$
k\left(\#_{n}(F)\right) \leq \#_{m}\left(F_{k}\right)<m(s-\varepsilon) .
$$

So,

$$
\bar{\mu}(F) \leq \lim _{n \rightarrow \infty} \frac{m(s-\varepsilon)}{k n}=\lim _{n \rightarrow \infty} \frac{\left(k n+l_{m}\right)(s-\varepsilon)}{k n}=s-\varepsilon \leq s .
$$

This contradicts to $\bar{\mu}(F) \geq s$.

This completes the proof.

\section{4. $\left(\mathcal{F}_{1}, \mathcal{F}_{2}\right)$-Chaos in Non-Autonomous Systems}

Now, we state the definition of $\left(\mathcal{F}_{1}, \mathcal{F}_{2}\right)$-chaos in nonautonomous systems.

Definition 2. Let $(X, \rho)$ be a compact metric space, $\mathcal{F}_{1}$ and $\mathcal{F}_{2}$ are two Furstenberg families. $\mathcal{D} \subset X$ is called $a\left(\mathcal{F}_{1}, \mathcal{F}_{2}\right)$-scrambled set of $\left(X, f_{1, \infty}\right)$ (briefly, $\left.f_{1, \infty}\right)$, if $\forall x \neq y \in \mathcal{D}$, the following two conditions are satisfied:

(i) $\forall t>0,\left\{n \in \mathbb{N}: \rho\left(f_{1}^{n}(x), f_{1}^{n}(y)\right)<t\right\} \in \mathcal{F}_{1}$;

(ii) $\exists \delta>0,\left\{n \in \mathbb{N}: \rho\left(f_{1}^{n}(x), f_{1}^{n}(y)\right)>\delta\right\} \in \mathcal{F}_{2}$.

The pair $(x, y)$ which satisfies the above two conditions is called an $\left(\mathcal{F}_{1}, \mathcal{F}_{2}\right)$-scrambled pair of $f_{1, \infty}$.

$f_{1, \infty}$ is said to be $\left(\mathcal{F}_{1}, \mathcal{F}_{2}\right)$-chaotic if there exists an uncountable $\left(\mathcal{F}_{1}, \mathcal{F}_{2}\right)$-scrambled set of $f_{1, \infty}$. If $\mathcal{F}_{1}=\mathcal{F}_{2}=\mathcal{F}, f_{1, \infty}$ is said to be $\mathcal{F}$-chaotic and $(x, y)$ is an $\mathcal{F}$-scrambled pair. $f_{1, \infty}$ is said to be strong $\left(\mathcal{F}_{1}, \mathcal{F}_{2}\right)$-chaotic if there are some $\delta>0$ and an uncountable subset $\mathcal{D} \subset X$ such that for any $x, y \in \mathcal{D}$ with $x \neq y$, the following two conditions holds:

(i) $\quad\left\{n \in \mathbb{N}: \rho\left(f_{1}^{n}(x), f_{1}^{n}(y)\right)<t\right\} \in \mathcal{F}_{1}$ for all $t>0$;

(ii) $\quad\left\{n \in \mathbb{N}: \rho\left(f_{1}^{n}(x), f_{1}^{n}(y)\right)>\delta\right\} \in \mathcal{F}_{2}$.

$f_{1, \infty}$ is said to be strong $\mathcal{F}$-chaos if it is strong $\left(\mathcal{F}_{1}, \mathcal{F}_{2}\right)$-chaotic and $\mathcal{F}_{1}=\mathcal{F}_{2}=\mathcal{F}$.

Let us recall the definitions of Li-Yorke chaos and distributional chaos in non-autonomous systems (see $[25,26])$. 
Definition 3. Assume that $\left(X, f_{1, \infty}\right)$ is a non-autonomous discrete system. If $x, y \in X$ with $x \neq y,(x, y)$ is called a Li-Yorke pair if

$$
\limsup _{n \rightarrow \infty} \rho\left(f_{1}^{n}(x), f_{1}^{n}(y)\right)>0 \text { and } \liminf _{n \rightarrow \infty} \rho\left(f_{1}^{n}(x), f_{1}^{n}(y)\right)=0 .
$$

The set $\mathcal{D} \subset X$ is called a Li-Yorke scrambled set if all points $x, y \in \mathcal{D}$ with $x \neq y,(x, y)$ is a Li-Yorke pair. $f_{1, \infty}$ is Li-Yorke chaotic if $X$ contains an uncountable Li-Yorke scrambled set.

Assume that $\left(X, f_{1, \infty}\right)$ is a non-autonomous discrete system. For any pair of points $x, y \in X$, define the upper and lower (distance) distributional functions generated by $f_{1, \infty}$ as

$$
F_{x y}^{*}\left(t, f_{1, \infty}\right)=\limsup _{n \rightarrow \infty} \frac{1}{n} \sum_{i=1}^{n} \chi_{[0, t)}\left(\rho\left(f_{1}^{i}(x), f_{1}^{i}(y)\right)\right)
$$

and

$$
F_{x y}\left(t, f_{1, \infty}\right)=\liminf _{n \rightarrow \infty} \frac{1}{n} \sum_{i=1}^{n} \chi_{[0, \delta)}\left(\rho\left(f_{1}^{i}(x), f_{1}^{i}(y)\right)\right)
$$

respectively. Where $\chi_{[0, t)}$ is the characteristic function of the set $[0, t)$, i.e., $\chi_{[0, t)}(a)=1$ when $a \in[0, t)$ or $\chi_{[0, t)}(a)=0$ when $a \notin[0, t)$.

Definition 4. $f_{1, \infty}$ is distributionally chaotic if exists an uncountable subset $D \subset X$ such that for any pair of distinct points $x, y \in D$, we have that $F_{x y}^{*}\left(t, f_{1, \infty}\right)=1$ for all $t>0$ and $F_{x y}\left(t, f_{1, \infty}\right)=0$ for some $\delta>0$.

The set $D$ is a distributionally scrambled set and the pair $(x, y)$ a distributionally chaotic pair.

It is not difficult to obtain that the pair $(x, y)$ is a $(\bar{M}(0), \bar{M}(0))$-scrambled pair if and only if $(x, y)$ is a Li-Yorke scrambled pair, and the pair $(x, y)$ is a $(\bar{M}(1), \bar{M}(1))$-scrambled pair if and only if $(x, y)$ is a distributionally scrambled pair. In fact,

$$
\bar{M}(0)=\mathcal{B}, \bar{M}(1)=\left\{F \in \mathcal{B}: \limsup _{n \rightarrow \infty} \frac{\#(F \cap\{1,2, \ldots, n\})}{n}=1\right\} .
$$

Then, $\left\{n \in \mathbb{N}: \rho\left(f_{1}^{n}(x), f_{1}^{n}(y)\right)<t\right\} \in \bar{M}(0)$ for any $t>0$ and $\left\{n \in \mathbb{N}: \rho\left(f_{1}^{n}(x), f_{1}^{n}(y)\right)>\delta\right\} \in$ $\bar{M}(0)$ for some $\delta>0$ is equivalent to that $\limsup _{n \rightarrow \infty} \rho\left(f_{1}^{n}(x), f_{1}^{n}(y)\right)>0$ and $\liminf _{n \rightarrow \infty} \rho\left(f_{1}^{n}(x), f_{1}^{n}(y)\right)=0$. $\left\{n \in \mathbb{N}: \rho\left(f_{1}^{n}(x), f_{1}^{n}(y)\right)<t\right\} \in \bar{M}(1)$ for any $t>0$ and $\left\{n \in \mathbb{N}: \rho\left(f_{1}^{n}(x), f_{1}^{n}(y)\right)>\delta\right\} \in \bar{M}(1)$ for some $\delta>0$ is equivalent to that $F_{x y}^{*}\left(t, f_{1, \infty}\right)=1$ and $F_{x y}\left(\delta, f_{1, \infty}\right)=0$.

Hence, $(\bar{M}(0), \bar{M}(0))$-chaos is Li-Yorke chaos and $(\bar{M}(1), \bar{M}(1))$-chaos is distributional Chaos.

\section{Main Results}

Theorem 1. Let $\mathcal{F}_{1}$ and $\mathcal{F}_{2}$ are two Furstenberg families with property $P(k)$, where $k$ is a positive integer. $\mathcal{F}_{1}$ is positive shift-invariant. If the system $\left(X, f_{1, \infty}\right)$ is $\left(\mathcal{F}_{1}, \mathcal{F}_{2}\right)$-chaos, then the system $\left(X, f_{1, \infty}^{[k]}\right)$ is $\left(\mathcal{F}_{1}, \mathcal{F}_{2}\right)$-chaos too.

Proof. If $D$ is an $\left(\mathcal{F}_{1}, \mathcal{F}_{2}\right)$-scrambled set of $f_{1, \infty}$, the following proves that $D$ is an $\left(\mathcal{F}_{1}, \mathcal{F}_{2}\right)$-scrambled set of $f_{1, \infty}^{[k]}$.

(i) Since $X$ is compact and $f_{i}(i \in \mathbb{N})$ are continuous, then, for any $j \in\{1,2, \ldots, k-1\}, f_{s_{1}}, \ldots, f_{s_{k-j}}$ are uniformly continuous (where $f_{s_{1}}, \ldots, f_{s_{k-j}}$ are freely chosen from the sequence $f_{i}(i \in \mathbb{N})$ ). That is, for any $\delta>0$, there exists a $\delta^{*}>0, \forall a, b \in X, \rho(a, b)<\delta^{*}$ implies $\rho\left(f_{s_{k-j}} \circ \cdots \circ\right.$ $\left.f_{s_{1}}(a), f_{s_{k-j}} \circ \cdots \circ f_{s_{1}}(b)\right)<\delta(j=1,2, \ldots, k-1)$. 
Since $D$ is an $\left(\mathcal{F}_{1}, \mathcal{F}_{2}\right)$-scrambled set of $f_{1, \infty}$, then, $\forall x \neq y \in D$, for the above $\delta^{*}$, we have

$$
F=\left\{n \in \mathbb{N}: \rho\left(f_{1}^{n}(x), f_{1}^{n}(y)\right)<\delta^{*}\right\} \in \mathcal{F}_{1} .
$$

And because $\mathcal{F}_{1}$ have property $P(k)$, there exists some $j \in\{1,2, \ldots, k-1\}$ such that

$$
F_{k, j}=\left\{i \in \mathbb{Z}^{+}: k i+j \in F\right\}=\left\{i \in \mathbb{Z}^{+}: \rho\left(f_{1}^{k i+j}(x), f_{1}^{k i+j}(y)\right)<\delta^{*}\right\} \in \mathcal{F}_{1} .
$$

By the selection of $\delta^{*}$, we put $s_{r}=k i+j+r(r=1,2, \ldots, k-j)$, then

$$
F_{k, j} \subset\left\{i \in \mathbb{Z}^{+}: \rho\left(f_{1}^{k i+j+k-j}(x), f_{1}^{k i+j+k-j}(y)\right)<\delta\right\}=\left\{i \in \mathbb{Z}^{+}: \rho\left(f_{1}^{k(i+1)}(x), f_{1}^{k(i+1)}(y)\right)<\delta\right\} .
$$

Write $F_{k, j}+1=\left\{i+1: i \in \mathbb{Z}^{+}, k i+j \in \mathcal{F}_{1}\right\}(\forall j=1,2, \ldots, k-1)$, then $F_{k, j}+1 \subset\left\{i \in \mathbb{Z}^{+}\right.$: $\left.\rho\left(f_{1}^{k i}(x), f_{1}^{k i}(y)\right)<\delta\right\}$.

By the positive shift-invariant of $\mathcal{F}_{1}$ and $F_{k, j} \in \mathcal{F}_{1}$, we have $F_{k, j}+1 \in \mathcal{F}_{1}$. And with the hereditary upwards of $\mathcal{F}_{1}$, for any $x, y \in D: x \neq y, \forall \delta>0,\left\{i \in \mathbb{Z}^{+}: \rho\left(f_{1}^{k i}(x), f_{1}^{k i}(y)\right)<\delta\right\} \in \mathcal{F}_{1}$.

(ii) Since $D$ is a $\left(\mathcal{F}_{1}, \mathcal{F}_{2}\right)$-scrambled set of $f_{1, \infty}$, then, for the above $x, y \in D(x \neq y), \exists \varepsilon^{*}>0$, such that $E=\left\{n \in \mathbb{Z}^{+}: \rho\left(f_{1}^{n}(x), f_{1}^{n}(y)\right)>\varepsilon^{*}\right\} \in \mathcal{F}_{2}$. And because $\mathcal{F}_{2}$ have property $P(k)$, then, there exists some $j \in\{1,2, \ldots, k-1\}$ such that

$$
E_{k, j}=\left\{i \in \mathbb{Z}^{+}: k i+j \in E\right\}=\left\{i \in \mathbb{Z}^{+}: \rho\left(f_{1}^{k i+j}(x), f_{1}^{k i+j}(y)\right)>\varepsilon^{*}\right\} \in \mathcal{F}_{2} .
$$

$X$ is compact and $f_{i}(i \in \mathbb{N})$ are continuous, then, for any $j \in\{1,2, \ldots, k-1\}, f_{s_{1}}, \ldots, f_{s_{j}}$ are uniformly continuous (where $f_{s_{1}}, \ldots, f_{s_{j}}$ are freely chosen from the sequence $f_{i}(i \in \mathbb{N})$ ). For the above $\varepsilon^{*}>0, \exists \varepsilon>0, \forall p, q \in X$ satisfied $\rho(p, q) \leq \varepsilon$, inequality $\rho\left(f_{s_{j}} \circ \cdots \circ f_{s_{1}}(p), f_{s_{j}} \circ \cdots \circ\right.$ $\left.f_{s_{1}}(q)\right) \leq \varepsilon^{*}$ holds.

The following will prove that $\left\{i \in \mathbb{Z}^{+}: \rho\left(f_{1}^{k i}(x), f_{1}^{k i}(y)\right)>\varepsilon\right\} \in \mathcal{F}_{2}$.

Suppose $\left\{i \in \mathbb{Z}^{+}: \rho\left(f_{1}^{k i}(x), f_{1}^{k i}(y)\right)>\varepsilon\right\} \notin \mathcal{F}_{2}$, then

$$
\mathbb{Z}^{+}-\left\{i \in \mathbb{Z}^{+}: \rho\left(f_{1}^{k i}(x), f_{1}^{k i}(y)\right)>\varepsilon\right\}=\left\{i \in \mathbb{Z}^{+}: \rho\left(f_{1}^{k i}(x), f_{1}^{k i}(y)\right) \leq \varepsilon\right\} \in k \mathcal{F}_{2} .
$$

By the selection of $\varepsilon^{*}$, we put $s_{r}=k i+r(r=1,2, \ldots, j)$, then

$$
\left\{i \in \mathbb{Z}^{+}: \rho\left(f_{1}^{k i+j}(x), f_{1}^{k i+j}(y)\right) \leq \varepsilon^{*}\right\} \in k \mathcal{F}_{2} .
$$

So,

$$
\left\{i \in \mathbb{Z}^{+}: \rho\left(f_{1}^{k i+j}(x), f_{1}^{k i+j}(y)\right)>\varepsilon^{*}\right\} \notin k \mathcal{F}_{2},
$$

This contradicts $E_{k, j} \in \mathcal{F}_{2}$.

Hence, for $x \neq y \in D$ in (i), there exists a $\varepsilon>0$ such that $\left\{i \in \mathbb{Z}^{+}: \rho\left(f_{1}^{k i}(x), f_{1}^{k i}(y)\right)>\varepsilon\right\} \in \mathcal{F}_{2}$.

Combining with (i) and (ii), $f_{1, \infty}^{[k]}$ is $\left(\mathcal{F}_{1}, \mathcal{F}_{2}\right)$-chaos.

This completes the proof.

Theorem 2. Let $\mathcal{F}_{1}$ and $\mathcal{F}_{2}$ are two Furstenberg families with property $Q(k)$, where $k$ is a positive integer. $\mathcal{F}_{2}$ is negative shift-invariant. If the system $\left(X, f_{1, \infty}^{[k]}\right)$ is $\left(\mathcal{F}_{1}, \mathcal{F}_{2}\right)$-chaos, then the system $\left(X, f_{1, \infty}\right)$ is $\left(\mathcal{F}_{1}, \mathcal{F}_{2}\right)$-chaos too.

Proof. If $D$ is a $\left(\mathcal{F}_{1}, \mathcal{F}_{2}\right)$-scrambled set of $f_{1, \infty}^{[k]}$, the following prove that $D$ is a $\left(\mathcal{F}_{1}, \mathcal{F}_{2}\right)$-scrambled set of $f_{1, \infty}$. 
(i) Similar to Theorem 1 , for any $j \in\{1,2, \ldots, k-1\}, f_{s_{1}}, \ldots, f_{s_{j}}$ are uniformly continuous (where $f_{s_{1}}, \ldots, f_{s_{j}}$ are freely chosen from the sequence $\left.f_{i}(i \in \mathbb{N})\right)$. That is, for any $\delta>0$, there exists a $\delta^{*}>0, \forall a, b \in X, \rho(a, b)<\delta^{*}$ implies $\rho\left(f_{s_{j}} \circ \cdots \circ f_{s_{1}}(a), f_{s_{j}} \circ \cdots \circ f_{s_{1}}(b)\right)<\delta(j=1,2, \ldots, k-1)$.

For any pair of distinct points $x, y \in D$, for the above $\delta^{*}$, one has

$$
F=\left\{n \in \mathbb{Z}^{+}: \rho\left(f_{1}^{k n}(x), f_{1}^{k n}(y)\right)<\delta^{*}\right\} \in \mathcal{F}_{1} .
$$

By the selection of $\delta^{*}$, for $\forall n \in F, \forall j \in\{1,2, \cdots, k-1\}$, put $s_{r}=k i+j+r(r=1,2, \ldots, j)$, then $\rho\left(f_{1}^{k n+j}(x), f_{1}^{k n+j}(y)\right)<\delta$. And because $\mathcal{F}_{1}$ have property $Q(k)$, then

$$
F_{k}=\left\{k n+j \in \mathbb{Z}^{+}: j=1,2, \ldots, k-1, n \in F\right\} \in \mathcal{F}_{1} .
$$

Notice that $F_{k} \subset\left\{m \in \mathbb{Z}^{+}: \rho\left(f_{1}^{m}(x), f_{1}^{m}(y)\right)<\delta\right\}$, then $\left\{m \in \mathbb{Z}^{+}: \rho\left(f_{1}^{m}(x), f_{1}^{m}(y)\right)<\delta\right\} \in \mathcal{F}_{1}$.

(ii) Since $D$ is an $\left(\mathcal{F}_{1}, \mathcal{F}_{2}\right)$-scrambled set of $f_{1, \infty}^{[k]}$, then, for the above $x, y \in D(x \neq y)$, there exist $\varepsilon^{*}>0$, such that $E=\left\{n \in \mathbb{Z}^{+}: \rho\left(f_{1}^{k n}(x), f_{1}^{k n}(y)\right)>\varepsilon^{*}\right\} \in \mathcal{F}_{2}$.

For any $j \in\{1,2, \ldots, k-1\}, f_{s_{1}}, \ldots, f_{s_{j}}$ are uniformly continuous (where $f_{s_{1}}, \ldots, f_{s_{j}}$ are freely chosen from the sequence $f_{i}(i \in \mathbb{N})$ ), then, for the above $\varepsilon^{*}>0$, there exist $\varepsilon>0$ such that $\rho(p, q)<\varepsilon(p, q \in X)$ implies $\rho\left(f_{s_{j}} \circ \cdots \circ f_{s_{1}}(p), f_{s_{j}} \circ \cdots \circ f_{s_{1}}(q)\right) \leq \varepsilon^{*}(j=1,2, \ldots, k-1)$. That is, $\rho\left(f_{1}^{k}(p), f_{1}^{k}(q)\right)>\varepsilon^{*}(p, q \in X)$ implies $\rho\left(f_{1}^{j}(p), f_{1}^{j}(q)\right)>\varepsilon(j=1,2, \ldots, k-1)$. $\forall n \in E, \forall j=1,2, \ldots, k-1$, put $s_{r}=k(n-1)+r(r=1,2, \ldots, j)$, then

$$
\rho\left(f_{1}^{k(n-1)+j}(x), f_{1}^{k(n-1)+j}(y)\right)>\varepsilon .
$$

Since $\mathcal{F}_{2}$ is negative shift-invariant, then $E-1 \in \mathcal{F}_{2}$. And because $\mathcal{F}_{2}$ have property $Q(k)$, then $(E-1)_{k} \in \mathcal{F}_{2}$, i.e., $\left\{k(n-1)+j \in \mathbb{Z}^{+}: n-1 \in E-1, j=1,2, \ldots, k-1\right\} \in \mathcal{F}_{2}$. Combining $(E-1)_{k} \subset\left\{m \in \mathbb{Z}^{+}: \rho\left(f_{1}^{m}(x), f_{1}^{m}(y)\right)>\varepsilon\right\}$ with the hereditary upwards of $\mathcal{F}_{2}$, we have $\left\{m \in \mathbb{Z}^{+}: \rho\left(f_{1}^{m}(x), f_{1}^{m}(y)\right)>\varepsilon\right\} \in \mathcal{F}_{2}$.

By (i) and (ii), $D$ is an $\left(\mathcal{F}_{1}, \mathcal{F}_{2}\right)$-scrambled set of $f_{1, \infty}$.

This completes the proof.

Similarly, the following corollaries hold.

Corollary 1. Let $\mathcal{F}_{1}$ and $\mathcal{F}_{2}$ are two Furstenberg families with property $P(k)$, where $k$ is a positive integer. $\mathcal{F}_{1}$ is positive shift-invariant. If the system $\left(X, f_{1, \infty}\right)$ is $\mathcal{F}$-chaos (strong $\left(\mathcal{F}_{1}, \mathcal{F}_{2}\right)$-chaos, or strong $\mathcal{F}$-chaos), then the system $\left(X, f_{1, \infty}^{[k]}\right)$ is $\mathcal{F}$-chaos (strong $\left(\mathcal{F}_{1}, \mathcal{F}_{2}\right)$-chaos, or strong $\mathcal{F}$-chaos).

Corollary 2. Let $\mathcal{F}_{1}$ and $\mathcal{F}_{2}$ are two Furstenberg families with property $Q(k)$, where $k$ is a positive integer. $\mathcal{F}_{2}$ is negative shift-invariant. If the system $\left(X, f_{1, \infty}^{[k]}\right)$ is $\mathcal{F}$-chaos (strong $\left(\mathcal{F}_{1}, \mathcal{F}_{2}\right)$-chaos, or strong $\mathcal{F}$-chaos), then the system $\left(X, f_{1, \infty}\right)$ is $\mathcal{F}$-chaos (strong $\left(\mathcal{F}_{1}, \mathcal{F}_{2}\right)$-chaos, or strong $\mathcal{F}$-chaos).

Combining with Propositions 1 and 2, Theorems 1 and 2, and Corollarys 1 and 2, the following conclusions are obtained.

Theorem 3. Let $s$ and t are arbitrary two numbers in $[0,1]$, then

(1) If $D$ is an $(\bar{M}(s), \bar{M}(t))$-scrambled set (or strong $(\bar{M}(s), \bar{M}(t))$-scrambled set) of $f_{1, \infty}$, then, for every $k \in \mathbb{Z}^{+}, D$ is an $(\bar{M}(s), \bar{M}(t))$-scrambled set(or strong $(\bar{M}(s), \bar{M}(t))$-scrambled set) of $f_{1, \infty}^{[k]}$.

(2) For some positive integer $k$, if $D$ is an $(\bar{M}(s), \bar{M}(t))$-scrambled set (or strong $(\bar{M}(s), \bar{M}(t))$-scrambled set) of $f_{1, \infty}^{[k]}$, then $D$ is an $(\bar{M}(s), \bar{M}(t))$-scrambled set (or strong $(\bar{M}(s), \bar{M}(t))$-scrambled set) of $f_{1, \infty}$. 


\section{Proof.}

(1) By Proposition 1, $\bar{M}(s)$ is shift-invariant (obviously positive shift-invariant). And because $\bar{M}(s), \bar{M}(t)$ are two Furstenberg families with property $P(k)$ (Proposition 2). Then, according to the proof of Theorem 1 , if $D$ is an $(\bar{M}(s), \bar{M}(t))$-scrambled set of $f_{1, \infty}$, then, for every $k \in \mathbb{Z}^{+}, D$ is an $(\bar{M}(s), \bar{M}(t))$-scrambled set of $f_{1, \infty}^{[k]}$.

(2) In the same way, (2) holds.

This completes the proof.

With the preparations in Section 4, we have

\section{Corollary 3.}

(1) If $D$ is a Li-Yorke scrambled set (or distributionally scrambled set) of $f_{1, \infty}$, then, for every $k \in \mathbb{Z}^{+}, D$ is a Li-Yorke scrambled set (or distributionally scrambled set) of $f_{1, \infty}^{[k]}$.

(2) For some positive integer $k$, if $D$ is a Li-Yorke scrambled set (or distributionally scrambled set) of $f_{1, \infty}^{[k]}$ then, $D$ is a Li-Yorke scrambled set (or distributionally scrambled set) of $f_{1, \infty}$.

Remark 1. In the non-autonomous systems, the iterative properties of Li-Yorke chaos and distributional chaos are discussed in $[25,26]$ before. The conclusions in Corollary 3 remains consistent with them.

This paper has presented several properties of $\left(\mathcal{F}_{1}, \mathcal{F}_{2}\right)$-chaos, strong $\left(\mathcal{F}_{1}, \mathcal{F}_{2}\right)$-chaos, and strong $\mathcal{F}$-chaos. There are some other problems, such as generically $\mathcal{F}$-chaos and $\mathcal{F}$-sensitivity, to discuss. Moreover, property $P(k)$ is closely related to congruence theory. Follow this line, one can consider other Furstenberg families which consist of number sets with some special characteristics.

Acknowledgments: This work was supported by National Natural Science Foundation of China (11501391, 61573010) and the Artificial Intelligence of Key Laboratory of Sichuan Province (2015RZJ01).

Author Contributions: Tianxiu Lu proposed the idea; Most of the conclusions are proved by Xiao Tang and Tianxiu Lu; Some important steps in Theorem 1 and Theorem 2 are inferred by Guanrong Chen; Tianxiu Lu wrote the paper.

Conflicts of Interest: The authors declare no conflict of interest.

\section{References}

1. Li, T.; Yorke, J. Period 3 implies chaos. Am. Math. Month. 1975, 82, 985-992.

2. Devaney, R.L. An Introduction to Chaotic Dynamical Systems; Addison Wesley: Boston, MA, USA, 1989.

3. Piorek, J. On generic chaos of shifts in funtion spaces. Ann. Polon. Math. 1990, 52, 139-146.

4. Snoha, L. Dense chaos. Comment. Math. Univ. Carolin. 1992, 33, 747-752.

5. Akin, E.; Kolyada, S. Li-Yorke sensitivity. Nonlinearity 2003, 16, 1421-1433.

6. Schweizer, B.; Smital, J. Measures of chaos and a spectral decomposition of dynamical systems on the interval. Trans. Am. Math. Soc. 1994, 344, 737-754.

7. Balibrea, F.; Smital, J. Strong distributional chaos and minimal sets. Topol. Appl. 2009, 156, 1673-1678.

8. Balibrea, F.; Smital, J.; Stefankova, M. On open problems concerning distributional chaos for triangular maps. Nonlinear Anal. Theor. 2011, 74, 7342-7346.

9. Li, R. A note on the three versions of distributional chaos. Commun. Nonlinear Sci. Numer. Simul. 2011, 16, 1993-1997.

10. Balibrea, F.; Smital, J.; Stefankova, M. Dynamical systems generating large sets of probability distribution functions. Chaos Soliton. Fract. 2014, 67, 38-42.

11. Wang, L.; Ou, X.; Gao, Y. A weakly mixing dynamical system with the whole space being a transitive extremal distributionally scrambled set. Chaos Soliton. Fract. 2015, 70, 130-133.

12. Shao, Y.; Gao, Y. The scrambling index set of primitive minimally strong digraphs. Linear Algebra Appl. 2016, $500,1-14$. 
13. Akin, E. Recurrence in Topological Dynamics: Furstenberg and Ellis Action; Plenum Press: New York, NY, USA, 1997.

14. Tan, F.; Zhang, R.F. On $\mathcal{F}$-sensitive pairs. Acta Math. Sci. 2011, 31, 1425-1435.

15. Wu, X.; Wang, J.; Chen, G. $\mathcal{F}$-sensitivity and Multi-sensitivity of hyperspatial dynamical systems. J. Math. Anal. Appl. 2015, 429, 16-26.

16. Wu, X.; Oprocha, P.; Chen, G. On various definitions of shadowing with average error in tracing. Nonlinearity 2016, 29, 1942-1972.

17. Kolyada, S.; Snoha, L. Topological entropy of non-autonomous dynamical systems. Random Comput. Dyn. 1996, 4, 205-233.

18. Elaydi, S.N. Non-autonomous difference equations: Open problems and conjectures. Fields Inst. Commum. 2004, 42, 423-428.

19. Elaydi, S.N.; Sacker, R.J. Non-autonomous Beverton-Holt equations and the Cushing-Henson conjectures. J. Differ. Equ. Appl. 2005, 11, 337-346.

20. Kolyada, S.; Snoha, L.; Trofimchuk, S. On minimality of non-autonomous dynamical systems. Neliniini Koliv. 2004, 7, 86-92.

21. Dvorakova, J. Chaos in non-autonomous discrete dynamical systems. Commun. Nonlinear Sci. Number. Simul. 2012, 17, 4649-4652.

22. Wu, X.; Ding, X.; Lu, T.; Wang, J. Topological dynamics of Zadeh's extension on upper semi-continuous fuzzy sets. Int. J. Bifurc. Chaos 2017, 27, 1750165.

23. Wu, X.; Wang, X.; Chen, G. On the large deviations theorem of weaker types. Int. J. Bifurc. Chaos 2017, 27, 1750127.

24. Li, Z.; Wang, H.; Xiong, J. Some remarks on $\mathcal{F}_{\infty}, \mathcal{F}_{\in}$-scrambled sets. ACTA Math. Sin. (Chin. Ser.) 2010, 53, 727-732.

25. $\mathrm{Wu}, \mathrm{X} . ; \mathrm{Zhu}, \mathrm{P}$. Chaos in a class of non-autonomous discrete systems. Appl. Math. Lett. 2013, 26, 431-436.

26. Lu, T.; Zhu, P.; Wu, X. Distributional chaos in non-autonomous discrete systems. Acta Math. Sci. (Chin. Ser.) 2015, 35, 558-566.

(C) 2018 by the authors. Licensee MDPI, Basel, Switzerland. This article is an open access article distributed under the terms and conditions of the Creative Commons Attribution (CC BY) license (http://creativecommons.org/licenses/by/4.0/). 